\title{
Arabidopsis NAP-related proteins (NRPs) contribute to the coordination of plant growth, developmental rate, and age-related pathogen resistance under short days
}

Balázs Barna ${ }^{3, *,}$, Katalin Gémes ${ }^{1,5, \S}$, Mónika Domoki ${ }^{1}$, Dóra Bernula ${ }^{1}$, Györgyi Ferenc ${ }^{1}$, Balázs Bálint ${ }^{4}$, István $\mathrm{Nagy}^{2,4}$, Attila Fehér ${ }^{1,5, *}$

${ }^{1}$ Institute of Plant Biology, and ${ }^{2}$ Institute of Biochemistry, Biological Research Centre of the Hungarian Academy of Sciences, Temesvári krt. 62., H-6726, Hungary

${ }^{3}$ Plant Protection Institute, Centre for Agricultural Research, Hungarian Academy of Sciences, Herman Ottó út 15, 1022 Budapest, Hungary

${ }^{4}$ SeqOmics Biotechnology Ltd, Vállalkozók útja 7, 6782 Mórahalom

${ }^{5}$ Department of Plant Biology, University of Szeged, Közép fasor 52, H-6726 Szeged, Hungary

*Corresponding author: Attila Fehér, Department of Plant Biology, University of Szeged, Közép fasor 52, H-6726 Szeged, Hungary, Szeged, Hungary; e-mail: feher.attila@brc.mta.hu $\S$ These two authors contributed equally to the results. 
This is the author's post-print version. The original containing supplementary information is avalable from Elsewire: https://doi.org/10.1016/j.plantsci.2017.11.006 
This is the author's post-print version. The original containing supplementary information is avalable from Elsewire: https://doi.org/10.1016/j.plantsci.2017.11.006

Conflicts of interest statement: The At NRP1 overexpressing plant line was produced by and provided for us for research purposes by the company CropDesign NV/SA Kerrebroek 66B; 9850 Nevele, Belgium. The nrp1-1 nrp2-1 mutant was produced by and provided for us for research purposes by Dr. Yan Zhu (State Key Laboratory of Genetic Engineering, Institute of Plant Biology, School of Sciences, Fudan University, Shanghai, China).

\section{Contributions:}

B.Barna contribution to research concept, plant pathology-related experiments, manuscript writing; K.G. developmental and gene expression studies, statistical data analysis, critical revision of the manuscript; M.D. molecular characterization of mutant/transgenic plants; D.B. plant cultures, sample preparation; G.F. RT-qPCR assay development; B.Bálint NGS data analysis; IN transcriptome sequencing; AF conception and experimental design, analysis and interpretation of the data, manuscript writing and finalization. 
This is the author's post-print version. The original containing supplementary information is avalable from Elsewire: https://doi.org/10.1016/i.plantsci.2017.11.006

\begin{abstract}
Plant nucleosome assembly protein-related proteins (NRPs) are histone chaperons involved in nucleosome turnover. Despite this basic cellular function, the Arabidopsis nrpl-1 nrp2-1 knock out mutant has been reported to exhibit only mild seedling root phenotypes and to significantly affect the expression of only few hundred genes [1]. Here we report that NRP loss-of-function as well as the ectopic overexpression of At NRP1 significantly affected the growth, development, and the pathogen response of Arabidopsis plants under short day conditions. The nrpl-1 nrp2-1 mutant grew faster and flowered weeks earlier than the wild type and the overexpressor. The latter developed slower and flowered at a lower number of leaves than the mutant and the wild type. Moreover, the mutant was more sensitive, the overexpressor was more tolerant to pathogen-induced necrosis correlating with their more adult and juvenile character, respectively. Transcriptomic comparison of mature non-bolting plants agreed with the phenotypes. The presented and other published data indicate that although NRPs might not be absolutely required for normal plant growth and development, their level needs to be controlled to allow the epigenetic coordination of metabolic, growth, defence and developmental processes during the acclimation to unfavourable growth conditions such as short days.
\end{abstract}

Key words: day length; disease tolerance, flowering time; histone chaperon; nucleosome assembly protein; plant age; 
This is the author's post-print version. The original containing supplementary information is avalable from Elsewire: https://doi.org/10.1016/i.plantsci.2017.11.006

\section{INTRODUCTION}

The nuclear genome of eukaryotes is structured by the help of specific proteins, the histones, resulting in the formation of the chromatin. The basic unit of this DNA-protein complex is the nucleosome, where 147 base pair of DNA is wrapped around a histone octamer consisting of two-two copies of $\mathrm{H} 2 \mathrm{~A}, \mathrm{H} 2 \mathrm{~B}, \mathrm{H} 3$, and $\mathrm{H} 4$, respectively [2]. The inter-nucleosomal DNA region is associated with $\mathrm{H} 1$ histones having role in the higher-order organization of the chromatin. In addition, all histones have variants increasing the structural and functional variability of nucleosomes. The genomic functions, including replication, repair, and transcription, largely depend on the accessibility of the DNA by various enzyme complexes. Therefore, the histone variants are in a continuous turnover that allows temporal and spatial reorganization of the overall nucleosome structure. This dynamic is, however, tightly controlled. Several covalent modifications of histones influence DNAhistone and histone-histone interactions and consequently the architecture and dynamics of the chromatin [3].

Histone chaperones control histone turnover. These acidic proteins binding to the positively charged histones facilitate their transport and have role in nucleosome assembly/disassembly determining the specificity of the DNA-histone interaction [4]. One of the families of histone chaperones includes the nucleosome assembly proteins (NAPs; [5]) sharing a structurally conserved fold, the NAP domain.

These proteins have been shown to have many functions in addition to serve as histone chaperones [5]. The family consists of the NAP1 protein and its close relatives as well as the structurally more distinct NAP-related SET (S.E. Translocation) protein having various other names due to its many functions (see e.g. in [6].

The NAP family was evolutionary conserved in all eukaryotes. Arabidopsis as well as rice have four NAP1 (NAP1; 1-4 also called NAPL1-4) and two NAP1-related proteins (NRP1 and 2 also called NAPL6 and 5) [7,8]. Plant NAP1 as well as NRP proteins were shown to bind histones H2A and H2B 
This is the author's post-print version. The original containing supplementary information is avalable from Elsewire: https://doi.org/10.1016/j.plantsci.2017.11.006

$[1,9,10]$ and are considered as histone $\mathrm{H} 2 \mathrm{~A}$ and $\mathrm{H} 2 \mathrm{~B}$ chaperones [8]. In accordance with their general function, the expression of the NAP1 as well as the NRP genes is rather ubiquitous in the Arabidopsis plant except NAP1; 4 that has a tissue specific expression in root segments and in the pollen grain [11]. The various proteins were found to have diverse intracellular distribution patterns depending on the investigated species, tissue, or physiological state as well as the used detection method [9-12]. While some members seem to shuttle between the cytoplasm and the nucleus, others seem to be mostly cytoplasmic or nuclear. Functional studies indicated that plant NAP1 proteins may be involved in the regulation of microtubule dynamics and the cell cycle [10], DNA repair [11], somatic homologous recombination [13,14], and the regulation of transcription [11] (for review see [8]). The NRP1 and 2 proteins have been implicated in post-embryogenic root growth, mitotic regulation, DNA repair, and transcription $[1,15,16]$ as well as somatic homologous recombination [14]. However, their role in these processes as well as the influenced genes seem to be distinct from those of the NAP1;1-4 proteins (e.g. $[8,11,14,16])$.

The plant NRP1 and NRP2 proteins fall into a phylogenetically distinct NAP1 subfamily also containing the animal SET and the yeast Vps75 proteins [7,8]. Both SET and Vps75 have several functions in association with various protein complexes (see [6,17], and the references therein). It is unknown how many of these interactions/functions are conserved in plants. In this regard, we have recently demonstrated that the Arabidopsis and Medicago NRP1 proteins have protein phosphatase 2A inhibitory activities similarly to their animal homologue, the SET protein [6]. Furthermore, GonzálezArzola and co-workers demonstrated that both the Arabidopsis NRP1 and the human SET (also called TAF-I $\beta$ ) proteins can interact with cytochrome $\mathrm{c}$ and control DNA repair in a similar manner [16].

Interestingly, despite of their basic cellular functions and the hundreds of affected genes [1,11], the loss of NAP1 and/or NRP functions were reported to exhibit mild or no phenotypes in Arabidopsis $[1,11]$. Although the nap 1;1 napl;2 napl;3 triple mutant exhibited increased sensitivity to various stresses, its growth and development were unaffected under normal conditions $[11,13]$. The nrpl-1 nrp 2-1 double mutant seedlings have short highly branched roots, but the mature plants have no any 
This is the author's post-print version. The original containing supplementary information is avalable from Elsewire: https://doi.org/10.1016/j.plantsci.2017.11.006

remarkable phenotype [1]. These plants exhibited, however, increased sensitivity against genotoxic stresses $[1,13]$. Even the sextuple mutant where all the six NAP1 family genes (including genes coding for the NRPs) had been deleted exhibited undisturbed growth and development under normal conditions [14].

Investigating the gene expression pattern of nrp1-1 nrp2-1 mutant seedlings, Zhu and coworkers [1] determined 102 genes that were differentially regulated in the double mutant in comparison to the wild type. In silico analysis of this gene set indicated that many of them might also be regulated by heat and/or pathogen infection [6]. Therefore, we decided to test the potential role of these proteins in heat shock as well as pathogen responses. In previous publications we have reported that although the overexpression of At NRP1 somewhat increased the heat tolerance [18], the NRPs were dispensable for heat-shock-induced gene expression in Arabidopsis [6]. To get a deeper insight into the reactions of the mutant and overexpressor plants to pathogens, we report here their response to artificial infections using the biotroph powdery mildew Golovinomyces orontii, the hemibiotroph Pseudomonas syringae bacteria, and the necrotroph Sclerotinia sclerotiorum fungus. In the pathological experiments, from practical points of view, plants grown at short day condition (11h light/13h dark) were used. It was observed that under this circumstance the NRP protein level strongly affected the growth, development, and senescence of Arabidopsis plants, as was indicated by plant size, flowering time, and photosynthetic pigment content. The effect of NRP protein absence and At NRP1 overexpression, respectively, on the growth, development, senescence, and pathogen sensitivity of short-day-grown (8h light 16h dark) Arabidopsis is also reported. Transcriptomic data obtained with mature, senescing plants grown at short days were used to strengthen the phenotypic observations including the pathogen responses. The potential role of NRPs as histone chaperones in the coordination of short-day responses of Arabidopsis plants is discussed.

\section{MATERIALS AND METHODS}


This is the author's post-print version. The original containing supplementary information is avalable from Elsewire: https://doi.org/10.1016/j.plantsci.2017.11.006

\subsection{Plants and pathogens}

Experiments were carried out with the wild-type Columbia ecotype of A. thaliana (L.) Heynh, its double mutant nrp1-1 nrp2-1 and overexpressing nrp1ox lines. The nrp1-1 nrp2-1 double mutant Arabidopsis plants are deficient in At NRP1 and At NRP2 expression and protein $[1,6]$. The seeds were kindly provided for us by Dr. Yan Zhu (State Key Laboratory of Genetic Engineering, Institute of Plant Biology, School of Sciences, Fudan University, Shanghai, China). The seeds of the At NRP1 genetic transformant (nrplox) approximately 2fold overexpressing the At NRP1 protein under the control of the sunflower GUbB1 ubiquitin promoter was kindly provided by Dr. Valerie Frankard (CropDesign N.V., Ghent, Belgium). The immunological detection of NRP protein levels in these and wild type plants is shown in the Supplementary material (Supplementary Fig. 1).

Seeds of Arabidopsis plants were sown into pots and kept 3 days at $5^{\circ} \mathrm{C}$ in dark, then put into a growth chamber (for the pathological experiments and pigment determination $11 \mathrm{~h}$ photoperiod light $/ 13 \mathrm{~h}$ dark, $140 \mu \mathrm{mol} / \mathrm{m} 2 / \mathrm{s}$, for the gene expression experiments $8 \mathrm{~h}$ photoperiod light $/ 16 \mathrm{~h}$ dark, $190 \mu \mathrm{mol} / \mathrm{m} 2 / \mathrm{s}$ ) with the temperature of $20^{\circ} \mathrm{C}$ and the plants were grown for the next 40 to 100 days depending on the type of the experiment.

The biotrophic powdery mildew (Golovinomyces orontii) strain MPIZ (kindly provided by Dr. Paul Schulze-Lefert, Max Planck Institute for Plant Breeding Research, Cologne, Germany) was used in the experiments. Strain MPIZ was maintained on Columbia ecotype of A. thaliana. Inoculation, spreading the conidia was carried out by touching the fully sporulating Arabidopsis leaves to the tested plants.

Two strains of the hemibiotrophic Pseudomonas bacteria were used: P. syringae pv. syringae 61 and P. syringae pv.tomato strain DC3000. These Pseudomonas strains are plant pathogens with differing host specificities and corresponding pathovar designations. P. syringae pv. 
This is the author's post-print version. The original containing supplementary information is avalable from Elsewire: https://doi.org/10.1016/j.plantsci.2017.11.006

syringae strain 61 elicits the hypersensitive response (HR) in nonhost plants..Pseudomonas syringae pv. tomato DC3000 (Pto DC3000) is the cause of bacterial speck disease on tomato and Arabidopsis, and gives a compatible reaction on Columbia ecotype. Bacterial cultures were maintained on nutrient agar at $30^{\circ} \mathrm{C}$. Cultures were transferred to fresh medium 16 to 24 $\mathrm{h}$ prior to use. Since we have evidence that even water injection itself can induce some membrane permeability changes, in the case of leakage measurements for infection all fully developed plant leaves were brushed either with water (control) or with bacterial suspension $\left(10^{8} \mathrm{cfu} \mathrm{cm}^{-3}\right)$. For measuring bacterial multiplication in plant tissue one centimetre diameter areas of fully expanded leaves in pre-flowering stage were infiltrated with bacterial suspensions or water as control using 1-ml needle-less syringe.

To have information on the effect of NRP protein on the reaction of plants to a typical necrotrophic pathogen infection with Sclerotinia sclerotiorum isolate Sz-24 (kindly provided by F. Virányi, Szent István University, Gödöllő, Hungary) was carried out as well. For one type of inoculation the fully developed leaves of the test plants were cut, placed on wet filter paper in Petri dishes and inoculated with 0.5 diameter agar culture disk of the pathogen. For leakage experiments, fully developed plant leaves were brushed either with water (control) or with Sclerotinia mycelial suspension. Evaluation of the symptoms was carried out by measuring lesion development on the leaves.

\subsection{Electrolyte leakage measurements}

Membrane damage was determined by measuring ion leakage from the leaves with an Oakton pH/conductivity meter (510 Series) according to the method described by [19]. Briefly, 5-5 whole control or infected wild type and mutant A. thaliana leaves were randomly taken from at least six - six plants, and placed on the surface of $15 \mathrm{ml}$ bi-distilled water Petri dishes. Ion 
This is the author's post-print version. The original containing supplementary information is avalable from Elsewire: https://doi.org/10.1016/j.plantsci.2017.11.006

leakage from leaves was measured periodically as changes in conductivity of the solution. The experiments were independently repeated five times with similar results.

\subsection{In planta bacterial multiplication}

Bacterial populations in leaf tissue were determined as described previously [20]. Briefly, $0.85-\mathrm{cm}$ diameter disk from leaf areas previously infiltrated with $10^{6}$ colony forming unit (cfu)/ml bacterial suspension was excised with a cork borer and ground in $1 \mathrm{ml}$ of $0.1 \mathrm{mM}$ $\mathrm{NaCl}$ in $25 \mathrm{mM}$ potassium phosphate buffer, $\mathrm{pH} 7.0$ with a mortar and pestle. The bacterial concentration was determined by dilution plating on nutrient agar plates. The experiments were independently repeated four times with similar results.

\subsection{Gene expression measurements by real-time quantitative PCR (RT-qPCR)}

Total RNA was extracted using Quick RNA ${ }^{\mathrm{TM}}$ Mini Prep kit (Zymo Research, Irvine, California, USA). The primers used are shown in the Supplementary material (Supplementary Table 1). One $\mu \mathrm{g}$ of total RNA from leaves was reverse-transcribed for $20 \mathrm{~min}$ at $42^{\circ} \mathrm{C}$ in a 20 $\mu 1$ reaction volume using Precision NanoScript 2 RT (Primerdesign Ltd. Chandler's Ford, United Kingdom) kit according to the manufacturer's instructions. The RT-qPCR reactions were carried out by the Applied Biosystems 7900-HT Fast Real-Time detection system (Thermo Fisher Scientific, Waltham, Massachusetts, USA). For amplification, a standard twostep thermal cycling profile was used $\left(15 \mathrm{sec}\right.$ at $95^{\circ} \mathrm{C}$ and $1 \mathrm{~min}$ at $\left.60^{\circ} \mathrm{C}\right)$ during 40 cycles, after a 10 -minute preheating step at $95^{\circ} \mathrm{C}$ ). Ct values were analysed using the RQ manager Software version 1.2 and then exported to Microsoft Excel for further analysis. The ratio of each mRNA relative to the AtGSTU1 was calculated using the 2- $\Delta \Delta C T$ method. The 
This is the author's post-print version. The original containing supplementary information is avalable from Elsewire: https://doi.org/10.1016/j.plantsci.2017.11.006

reference gene AtGSTU1 (Arabidopsis thaliana glutathione S-transferase TAU 1;

AT2G29490) was selected based on its uniform expression in the transcriptomic analysis (see further). Two biological and three technical replicates were analysed.

\subsection{Statistical analysis and data representation}

Sample/repetition numbers are given at the respective experimental description. Statistical evaluations were performed using the SigmaPlot v.12.0 statistical software. Quantitative data are presented as the mean \pm SEM and the significance of difference between sets of data was determined by one-way analysis of variance (ANOVA) following Duncan's multiple range tests; a P value of less than 0.05 was considered significant.

\subsection{Transcriptome sequencing and data analysis}

Whole transcriptome sequencing was performed using TrueSeq RNA Library Preparation Kit v2 (Illumina, San Diego, California, USA) according to the manufacturer's instructions. The duplicated samples derived from two independent experiments. Briefly, RNA quality and quantity measurements were performed using RNA ScreenTape and Reagents on TapeStation (all from Agilent, Santa Clara, California, USA) and Qubit (Thermo Fisher); only high quality (RIN >8.0) total RNA samples were processed. Next, RNA was DNaseI (Thermo Fisher) treated and the mRNA was purified and fragmented. First strand cDNA synthesis was performed using SuperScript II (Thermo Fisher) followed by second strand cDNA synthesis, end repair, 3'-end adenylation, adapter ligation and PCR amplification. All the purification steps were performed using AmPureXP Beads (Backman Coulter, Brea, California, USA). Final libraries were quality checked using D1000 ScreenTape and Reagents on TapeStation (all from Agilent). Concentration of each library was determined using the QPCR NGS 
This is the author's post-print version. The original containing supplementary information is avalable from Elsewire: https://doi.org/10.1016/j.plantsci.2017.11.006

Quantification Kit for Illumina (Agilent). Sequencing was performed on Illumina instrument using the HiSeq SBS Kit v4 250 cycles kit (Illumina) generating >20 million clusters for each sample.

Data availability: Gene Expression Omnibus (GEO) archive of the sequenced libraries was deposited in NCBI's GEO Archive at http://www.ncbi.nlm.nih.gov/geo under accession GSE99982.

Bioinformatic analysis: Raw data quality assessment, read trimming (Phred quality threshold 13), read mapping and gene expression profiling was carried out in CLC Genomics Workbench tool (CLC Bio now part of Qiagen, version 8.5.1) using Arabidopsis thaliana genome assembly “THAIR10" as a mapping reference. Only those reads that displayed at least $80 \%$ sequence identity against the reference over at least $80 \%$ of their lengths were mapped. Repeat reads with up to 30 possible genomic locations were randomly placed. Extremely repeated reads (reads with more than 30 possible genomic locations) were excluded.

“Total gene read" RNA-Seq count data was imported from CLC into R version 3.2.0 . Subsequently, "calcNormFactors" from package "edgeR" version 3.2.2 [21] was used to perform data normalization based on the "trimmed mean of M-values" (TMM) method [22]. Log transformation was carried out by the "voom" function of the "limma" package version 3.18.13 [23]. List of differentially expressed genes in each comparison was then generated with edgeR functions "exactTest" and "topTags"; genes showing at least two-fold gene expression change with an FDR value below 0.05 were considered as significant. Volcano plots showing statistically significant gene expression changes were also obtained in R.

Gene ontology (GO) analysis was made with the parametric gene set enrichment analysis tool of the agriGo ([24]; http://bioinfo.cau.edu.cn/agriGo/) toolkit. Only the genes with significant 
This is the author's post-print version. The original containing supplementary information is avalable from Elsewire: https://doi.org/10.1016/j.plantsci.2017.11.006

change in gene expression (see before) were considered in the analysis using the Plant GO Slim ontology type.

\section{RESULTS}

\subsection{The NRP level affects pathogen infection and symptom development of plants}

To investigate the effect of NRP proteins on the pathogen response, the nrpl-1 nrp2-1 double T-DNA insertion mutant (nrpmut) and the At NRP1 overexpressing nrplox Arabidopsis plants were infected by biotrophic (powdery mildew, Golovinomyces orontii MPIZ strain) and necrotrophic (Sclerotinia sclerotiorum) fungal pathogens. For the pathogen response tests, the plants were cultured under short day (11h light/13h dark) conditions. Four-five days after infection with powdery mildew, the first symptoms appeared on the tested Arabidopsis plants. All the three investigated genotypes were susceptible; the sporulation was the weakest on the nrpmut plant, and the strongest on overexpressor leaves. To get quantitative data, the ion leakage from the control and infected leaves was determined. The biotrophic powdery mildew did not induce strong ion leakage even seven days after infection when the symptoms fully developed (Fig. 1A). Nevertheless, the leakage experiments agreed with the symptoms. The measurements were carried out at later stage of plant development, because powdery mildew infection needs 5-7 days for full sporulation. At this developmental state, the nrpmut leaves exhibited stronger leakage of electrolytes even without infection, which was probably due to their faster senescence, while uninfected nrplox leaves showed less leakage as compared to that of the wild type, although this difference was not significant (Fig. 1A). The only significant increase of ion leakage in response to infection was measured in the case of this line (Fig. 1A), probably as a consequence of the densest mildew infection.

Inoculation of leaves with the necrotroph Sclerotinia resulted in large necrotic flecks, which was the largest on the NRP knockout mutant, and the smallest on the At NRP1 overexpressor leaves (Fig. 1B,C). Infection with the necrotrophic pathogen Sclerotinia resulted in a strong leakage of ions $96 \mathrm{~h}$ 
This is the author's post-print version. The original containing supplementary information is avalable from Elsewire: https://doi.org/10.1016/j.plantsci.2017.11.006

after inoculation of the investigated Arabidopsis plants. The significantly $(\mathrm{P} \leq 0,05)$ largest increase of ion leakage was measured from the nrpmut and the smallest from nrplox leaves (Fig.1D).

The response of the plant lines towards bacterial infection was also tested. The virulent $P$. syringae pv. tomato DC 3000 (DC 3000) started to induce disease symptoms (yellow flecks) on all Arabidopsis lines already 3 days after brushing the leaves with bacterial suspension. The most severe symptoms appeared on the nrpmut plants. None of the three genotypes showed visible symptoms inoculating the leaves with the avirulent $P$. syringae pv. syringae 61 (Ps 61) bacteria using the same conditions. Ion leakage from control or bacteria-infected leaves were measured to quantify the differences in tissue damage (Fig. 2A). Infection with both the virulent and the avirulent strains increased the leakage of electrolytes from the tested leaves 72 hours after infection reflecting the symptom development and the hypersensitive response, respectively. The largest membrane damage was measured from the nrpmut leaves with both types of the tested bacteria. The wild type and the nrplox plants exhibited similar responses.

The resistance of the tested plants was also investigated by measuring bacterial multiplication. The results showed that 72 hours after inoculation the number of colony forming units of the virulent DC 3000 bacteria increased in all cases, in agreement with its compatibility. The largest number of virulent bacteria was measured in the leaves of the At NRP1 overexpressor plants (Fig. 2B).

\subsection{The effect of altered NRP levels on the growth and development of Arabidopsis plants under short day conditions}

For the pathogen response tests, the plants were cultured under short day (11h light/13h dark) conditions allowing the development of large rosettes to facilitate infection and evaluation. It could be easily recognised that the nrpmut line had a faster growth rate and earlier senescence under this condition (Supplementary Fig. 2). According to our and others earlier observations $[1,6,18]$, the mutant and overexpressor plants did not show observable growth phenotype under long-day 
This is the author's post-print version. The original containing supplementary information is avalable from Elsewire: https://doi.org/10.1016/j.plantsci.2017.11.006

conditions. Therefore, detailed characterization of short-day-grown plants ( $8 \mathrm{~h}$ light/16h dark) have been carried out comparing the nrp1-1 nrp2-1 null mutants to At NRP1 overexpressor and wild type plants.

Under these conditions the nrpmut plants grew much faster reaching more than double the rosette size of the wild type or nrplox lines at around four weeks (Fig. 3). Not only the growth but the development of the mutant line has also been accelerated. The mutant plants reached a higher leaf number and flowered earlier but their bolting started approximately at the same leaf number then that of the wild type (Fig. 4A,B). The overexpressor plants flowered at a significantly lower leaf number than the control plants but required a much longer time to develop this number of leaves (Fig. 4A). In addition to flowering, the senescence of the mutant plants has started earlier as could be determined visually (Fig. 4B,C). In contrast, the senescence of the leaves of the nrplox plants was slightly delayed as compared to the wild type (Fig. 4B,C).

\subsection{The NRP level influences the transcriptome of short-day-grown mature plants}

In order to determine whether the developmental effects of the NRP proteins is related to transcriptional regulation, leaf samples were collected from 72-day-old short-day-grown plantlets, just before the nrpmut plants started bolting. Two biologically independent samples per plant line were subjected to mRNA isolation and high-throughput transcriptome sequencing (RNA-Seq) (see Supplementary Fig. 3 for a heat map). Transcript abundance of the wild type leaves was compared pairwise to that of the nrpmut and the nrplox transgenic lines. Altogether, the transcript abundance of 1799 genes exhibited at least two-fold changes in a statistically significant manner (FDR value below 0.05): 450 (25\%) in the nrpmut; 1005 (56\%) in the nrplox line; 344 in both (19\%) (see Supplementary Table 2 for details). 278 genes $(15,4 \%)$ changed their expression in the same direction and only $63(3,5 \%)$ genes were upregulated in the mutant and downregulated in the overexpressor while the opposite 
This is the author's post-print version. The original containing supplementary information is avalable from Elsewire: https://doi.org/10.1016/j.plantsci.2017.11.006

happened only in 3 cases $(0,2 \%)$ (Fig. 5). Even though only $19 \%$ of the regulated individual genes were common in the mutant and the overexpressor, the functional annotation of transcripts indicated that the down or upregulation of NRP protein level affected the same processes. Functional groups of genes were regulated either in the same or the opposite way in the mutant and overexpressor lines in correlation with the observed phenotypes (Tables 1 and 2). The annotation of transcripts indicated that the genes upregulated in overexpressor plants are connected to response to stress (174 genes), to response to biotic stimulus (59 genes) and to response to stimulus (287 genes), while the same type of genes are downregulated in the mutant plants (86 genes, 30 genes and 134 genes, respectively). In contrast, the genes connected to reproduction are upregulated (reproductive structure development 17 genes, reproduction 20 genes, reproductive process 20 genes and reproductive developmental process 18 genes) only in the mutant plants in correlation with their earlier flowering as compared to the wild type and overexpressor plants (Tables 1 and 2). Gene ontology enrichment in relation to catabolic processes as well as the under representation of transcripts associated with cell differentiation and the regulation of cellular processes in the nrpmut leaves well correlates with the early senescence of these plants (Table 1). Genes affecting multi-organism processes and biotic interactions were under represented in the mutant in agreement with their elevated sensitivity towards pathogenic fungi. In contrast, GO enrichment in the gene set regulated in the nrplox plants indicated the significant over representation of genes to biotic stimulus and to multi-organism processes underlying their elevated tolerance to pathogen-induced necrosis. It is noteworthy that genes connected to cellular metabolic processes, cellular process, cellular developmental process and cell growth are down regulated in nrplox Arabidopsis in correlation with the slow growth and development of these plants (Table 2). The increased catabolism and secondary 
This is the author's post-print version. The original containing supplementary information is avalable from Elsewire: https://doi.org/10.1016/j.plantsci.2017.11.006

metabolism, the down-regulated energy production and photosynthesis might also be related to their slow development.

The expression pattern of genes associated with senescence and pathogen response (coding for the WRKY transcription factors WRKY 30, 40, 45, the senescence-associated protein SAG13, and the pathogenesis-related proteins PR1, PR4, PR5) has also been determined by RT-qPCR in parallel mRNA samples as used in the transcriptomic approach. The relative expression levels obtained by RT-qPCR correlated well with the transcript abundance ratios determined by RNA sequencing (Fig. 6).

\section{DISCUSSION}

\subsection{The function of NRPs and their role in plant growth and development}

The chromatin has a dynamic structure that allows the regulation of DNA functions including replication, repair, and transcription [25]. The basic unit of the chromatin is the nucleosome, where 147 base pair of DNA is wrapped around a histone octamer [2]. Removal, recycling, or exchange of histones during nucleosome turnover is required to maintain epigenetic stability preserving homeostasis as well as to reorganise the chromatin ensuring dynamic cellular responses and adaptation [26]. Chromatin structure is controlled at several levels including the methylation of the DNA, the various post-transcriptional modifications of histones, the action of chromatin remodelling ATPases, and histone chaperones [27]. Nucleosome assembly proteins (NAPs) and their relatives (NRPs) form an evolutionary conserved family of histone chaperones having a primary role in nucleosome turnover [5].

Despite their general function, loss-of-function of Arabidopsis NAP and NAP-related protein (NRP) genes doesn't result in serious growth or developmental alterations under normal growth conditions unlike that of certain animal counterparts (for review, [8]). Even 
This is the author's post-print version. The original containing supplementary information is avalable from Elsewire: https://doi.org/10.1016/j.plantsci.2017.11.006

the triple mutant of the three canonical Arabidopsis NAP1 genes (NAP1;1, NAP1;2 and NAP1;3) showed wild-type plant growth and development under standard laboratory growth conditions [11]. Similarly, the nrp1-1 nrp2-1 double mutant missing the function of both Arabidopsis NRP genes exhibited only a mild seedling root phenotype: short branching root with disordered cellular organization due to arrested cell-cycle progression at the G2/M phase transition [1]. The growth and development of mature plants, however, was not affected by the absence of NRPs. Therefore, neither NAP nor NRP proteins seem to be indispensable for mature plant growth and morphogenesis under normal conditions. In this paper, we provide evidence that NRPs significantly interfere with Arabidopsis growth, development, and pathogen sensitivity under short day conditions.

\subsection{The level of NRPs influences the ageing of Arabidopsis plants}

The NRP loss-of-function mutant plants exhibited senescence symptoms earlier than the wild type, and it was the opposite in the case of the At NRP1 overproducers. Among the 6323 genes identified as differentially expressed during Arabidopsis leaf senescence [34], 644 were significantly deregulated in either the nrpmut or the nrplox plants in comparison to the wild type (Supplementary Table 3). In contrast to the observed phenotypic difference, the transcriptomic data indicated that the nrplox plants also went through senescence at the investigated time: e.g. photosynthesis was downregulated, catabolic processes and secondary metabolism were upregulated in the overexpressor in comparison to the wild type (Table 2). Moreover, the senescence-associated gene set was more strongly upregulated in the overexpressor than in the mutant, however, the number of downregulated genes was also higher (Supplementary Table 3). A possible explanation for the contradiction between the senescence symptoms and the transcriptomic data can be the developmental shift among the 
This is the author's post-print version. The original containing supplementary information is avalable from Elsewire: https://doi.org/10.1016/j.plantsci.2017.11.006

compared plant lines at the sampling time (e.g. the wild type did not yet reach, while the nrpmut plants had already passed the senescence state of the nrplox plants at 72 days)( see e.g. [34]). One can also hypothesize that the upregulation of genes associated with stress tolerance and defence reactions may also account for the delayed symptom development in the nrplox plants (Table 2).

\subsection{The level of NRPs influences pathogen response and symptom development under short day conditions}

Short day conditions, developmental plant age, as well as the redox status are all known to influence the pathogen response of plants. This agrees with the observed pathogen response of the late senescing nrplox and early senescing nrpmut plant lines. The necrotrophic pathogen Sclerotinia caused the largest damage on the early-senescing mutant plants, and the smallest on the overproducing plants with delayed senescence (Fig. 1). The elevated susceptibility of older plant tissues is probably connected to their augmented sensitivity to toxins, to cell-wall degrading enzymes, to reactive oxygen species, as well as to more intensive cell membrane lipid degradation $[19,35]$. All the three genotypes were susceptible to powdery mildew, and no hypersensitive type of resistance, just sporulation was seen by naked eyes. The low increase of leaked ions reflected that the biotrophic powdery mildew did not cause too much damage to the plant membranes under the applied conditions. The sporulation was the weakest on the nrpmut, and the strongest on the nrplox leaves, which harmonizes the idea, that biotrophic pathogens prefer juvenile plant tissues [36,37].

The largest tissue damage caused by the hemibiotrophic Pseudomonas bacteria was found in the case of the early senescing $n r p 1-1$ nrp2-1 double mutant plants. The avirulent $P$. syringae pv. syringae 61 caused cellular damage by induction of hypersensitive response 
This is the author's post-print version. The original containing supplementary information is avalable from Elsewire: https://doi.org/10.1016/j.plantsci.2017.11.006

while the virulent $P$. syringae pv.tomato strain DC3000 developed the disease on the leaves resulting in ion leakage (Fig. 2). The multiplication of the avirulent bacteria was inhibited early and there were no significant differences in the three plant genotypes, but the virulent bacteria multiplied vigorously, and the largest bacterial concentration was measured in the late-senescing At NRP1 overproducer plants (Fig. 2). These data agree with previous results when senescence was inhibited in cytokinin-overproducing transgenic tobacco lines [20]. In those lines, the avirulent $P$. syringae pv. tomato bacteria-induced hypersensitive response was suppressed, but the multiplication of the virulent $P$. syringae pv. tabaci was significantly elevated [20].

The pathogen infection tests are in accordance with the hypothesis, that senescence of plant tissues is favourable to necrotrophic, while juvenility is generally favourable to biotrophic pathogens [36-38].

\subsection{The level of NRPs influences the flowering time of Arabidopsis under short days}

Arabidopsis is a facultative long-day plant developing faster and flowering much earlier under long-day than short-day conditions. It was observed that the nrp1-1 nrp2-1 double mutant line (nrpmut) flowered 1-3 weeks earlier under short days than the wild type both having around the same leaf number, while the At NRP1 overexpressing nprlox plants flowered at around the same time then the wild type but with a lower leaf number (Fig. 4). These observations indicate that NRPs have role in coupling of leaf number with flowering time under short days.

Environmental and developmental factors regulating the flowering response of Arabidopsis converge on the so-called floral integrators FLOWERING LOCUS T (FT), SUPRESSOR OF OVEREXPRESSION OF CONSTANS 1 (SOC1) and LEAFY (LFY) (e.g. 
This is the author's post-print version. The original containing supplementary information is avalable from Elsewire: https://doi.org/10.1016/j.plantsci.2017.11.006

[28]). While under long-days these factors are activated due to the light-dependent accumulation of the CONSTANS (CO) protein in the leaves inducing FT and consequently SOC1 and LFY expression, under short days, the plant hormone gibberellic acid (GA) takes over this regulatory role [28]. GA regulates flowering under short days via the SOC1-SPL (SPL stands for SQUAMOSA PROMOTER BINDING PROTEIN-LIKE) module [29]. The SPL3,4,5 genes integrate endogenous ageing signals with GA action during the induction of flowering in short days that is dependent on SOC1 expression [29,30]. The transcriptomic data of yet non-bolting 72-days-old plants indicated that the expressions of neither the SPL, nor the SOC1, nor other flowering integrator genes were significantly altered in the nrpmut or nrplox plants (see Supplementary Table 2). This may indicate that either the vegetative-toreproductive transition was not yet initiated at this stage or the effect of NRPs on flowering is independent on the above pathways. This latter possibility is supported by the number of genes associated with reproduction or reproductive structure development that are upregulated in the mutant in comparison to the wild type (Table 1). This gene set includes the known flowering regulator FLOWERING PROMOTING FACTOR1 (FPF1) gene (AT5G24860). This gene was found to be significantly (approximately twenty-fold) upregulated in both the nrpmut and nrplox plants as compared to the wild type.

FPF1 is a small $12,6 \mathrm{kD}$ protein the overexpression of which promotes the floral transition via the modulation of the GA response in the apical meristem [31,32]. Interestingly, the FPF1 overproduction also resulted in larger rosette leaves and shortened juvenile phase of the transgenic plants [32]. Genetic interaction experiments showed that FPF1 acts in parallel with the day-length-dependent and the autonomous flowering pathways in agreement with the fact that its overexpression promotes flowering under long as well as short days. Since FPF1 
This is the author's post-print version. The original containing supplementary information is avalable from Elsewire: https://doi.org/10.1016/j.plantsci.2017.11.006

was upregulated in both the NRP mutant and the At NRP1 overexpressor, its action might depend on other factors that are differentially affected by the NRP level.

Recently, a mutation in another chromatin modifying gene coding for the JmjC DOMAIN-CONTAINING PROTEIN 27 (JMJ27), a histone demethylase, has been found to affect the flowering of Arabidopsis in a similar day-length dependent manner, although in that case it could be correlated with the increased expressions of the FLC, SOC1, and FT genes [33]. These observations support the importance of chromatin organisation in the coordination of flowering time with day length.

\subsection{NRPs might have role in the coordination of responses to short day conditions}

The light period has been shown to be interlinked with the antioxidative capacities, the pathogen response, the flowering time, and the life span of Arabidopsis [39,40]. Short day conditions are known to influence the redox balance of plant cells via the increased production of reactive oxygen species and the altered responses to it [39-44]. Under these conditions, the redox-mediated regulation of the system preventing oxidative damages under long days is overridden to allow more efficient light usage [39]. The cellular metabolism and the redox status are known to directly affect the activity of various chromatin modifying enzymes [45]. These enzymes are likely involved in the epigenetic coordination of gene expression that is required for the acclimation to short day conditions. Both in the nrpmut and nrplox plants several developmental and metabolic processes were found to be deregulated at the gene expression level indicating the loss of this coordination (Tables 1 and 2). NRPs as histone chaperons might indirectly be involved in the acclimation process controlling nucleosome dynamics. The level of NRPs is not influenced by day length (see Supplementary Fig. 4). This agrees with the observation that both low and high NRP levels interfere with the 
This is the author's post-print version. The original containing supplementary information is avalable from Elsewire: https://doi.org/10.1016/j.plantsci.2017.11.006

adaptation to short day conditions. It also indicates that NRPs might be post-translationally regulated during the process.

A potential link of NRPs to redox regulation might also depend on their PP2A phosphatase inhibitor activity [6]. PP2A phosphatases are involved in many cellular processes including ROS signalling [46]. The specific activity of a PP2A phosphatase complex depends on regulatory subunits. The PP2A B' $\gamma$ subunit was shown to be an important player in controlling flowering time [47] as well as short-day-dependent intracellular oxidative stress and pathogen responses [40]. PP2A B' $\gamma$ likely affects flowering regulation contributing to the repression of FLOWERING LOCUS C (FLC) [47], and have role in the regulation of senescence and pathogen-responses via the activation of CONSTITUTIVE EXPRESSION OF PR GENES5 (CPR5) [40,48], however, neither of these genes seem to be directly affected by the NRP level according to our transcriptomic data.

It is known that older leaves/plants display increased resistance or reduced susceptibility to certain pathogens that is often referred as age-related resistance (ARR) (discussed in detail in [49]). The production of defence-associated compounds in un-infected old leaves/plants supports the developmental regulation of ARR. Among others, pathogenesis-related (PR) and PR-like genes were shown to be upregulated during flower development and senescence [49]. Our transcriptomic data indicated that the pathogendefence related gene set (with gene ontologies related to biotic interactions, biotic stimulus, multi-organismal processes) was down regulated in the nrpmut and upregulated in the nrplox plants (Tables 1-2). This NRP-controlled gene set included among others those coding for the pathogenesis-related proteins PR1, PR4, PR5 proteins, a basic chitinase, and a beta 1.3glucanase (Supplementary Table 4). The fact that despite their faster senesce the nrpmut plants have lower PR gene expression and are more sensitive towards bacterial pathogens 
This is the author's post-print version. The original containing supplementary information is avalable from Elsewire: https://doi.org/10.1016/j.plantsci.2017.11.006

suggests that NRPs are required for ARR that can be uncoupled from developmental plant age (i.e. earlier flowering and senescence). Recently, it has been shown that the chromatin modifying histone demethylase JmjC DOMAIN-CONTAINING PROTEIN 27 (JMJ27) similarly affected flowering time and PR gene expression under short days [33] than the NRPs. These observations further support the central role of the chromatin in the coordination of development and defence that is more pronounced under short- than long-day conditions.

NAPs as well as NRPs have already been shown to play roles in plant responses to changing environmental conditions. Loss-of-function triple mutant of the Arabidopsis nap 1-1 nap1-2 nap1-3 genes exhibited hyper-sensitivity to genotoxic and salt stress treatments as well as to phosphate starvation $[11,13,50]$. Arabidopsis NRP1 and NRP2 were also shown to have role in genotoxic stress tolerance, DNA repair, and the maintenance of genome integrity $[1,13,16]$. Our preliminary observations indicated that the At NRP1-overexpressing mature plants can tolerate drought much better than the wild type or the nrpl-1 nrp2-1 mutant (B. Barna; unpublished), in agreement with the upregulation of many stress response genes in the nrplox background (Table 2.). NRPs, therefore, might be generally involved in the reorganization of gene expression during the adaptation to stress conditions including short days.

\subsection{The regulation of gene expression by NRPs}

The histone chaperon activity of NRPs has been proven in vitro as well as in vivo $[1,15,16]$. Arabidopsis NRP1 proteins were shown to be recruited to a specific promoter region (GLABRA2) by a transcription factor (WEREWOLF) where they promoted histone eviction and nucleosome loss [15]. Furthermore, the NRP1-histone interaction facilitated the transcription factor-to-DNA interaction. At present, it is unclear how much this regulatory 
This is the author's post-print version. The original containing supplementary information is avalable from Elsewire: https://doi.org/10.1016/j.plantsci.2017.11.006

model described for root hair development can be generalized. It is likely that NRPs may regulate gene expression in various ways since they are required for chromatin-mediated gene silencing [1] as well as for nucleosome loss-mediated gene activation [15].

Pathogenesis-related (PR) gene expression is regulated by WRKY transcription factors and basic leucine zipper proteins of the TGA family (see e.g. in [1]). Among these, several WRKYs were found to be upregulated in the nrplox plants (Fig. 6; Supplementary Table 4) that may contribute to their increased pathogen tolerance. In addition to the above TFs, PR genes are under the control of the positive coregulator NONEXPRESSOR OF PR GENES1 (NPR1), and the repressor SUPPRESSOR OF NPR1, INDUCIBLE1 (SNI1) [51]. In response to pathogen infection, salicylic acid promotes the translocation of NPR1 to the nucleus where it serves as a cofactor of TGA transcription factors. NPR1 is also proposed to inactivate the transcriptional repressor SUPPRESSOR OF NPR1, INDUCIBLE1 (SNI1). In the snil single mutant, the NPR1-dependent PR genes are specifically derepressed due to chromatin modifications at the gene promoters [52]. SNI1 is a scaffold protein likely recruiting chromatin remodelling factors to the promoters. Among others, SNI1 acts via RAD51D the activity of which makes the chromosome more accessible for transcription and homologous recombination [53]. NRPs are involved in both of these processes (i.e. transcriptional regulation and homologous recombination) [13]. It is tempting to speculate that NRPs act on pathogenesis-related gene expression via the modulation of the chromatin remodelling functions of SNI1/RAD51D. Since the expression of none of the above factors (TGA TFs, NPR1, SNI1, RAD51D) was influenced by the NRP level in the transgenic plants, this modulation might be exerted at the protein-protein or protein-chromatin interaction level.

The overall gene set significantly deregulated in the nrpmut and nrplox backgrounds showed only a limited overlap (Fig. 5; Supplementary Table 2). Very often both the increased 
This is the author's post-print version. The original containing supplementary information is avalable from Elsewire: https://doi.org/10.1016/j.plantsci.2017.11.006

and the decreased NRP(s) level affected gene expression in a similar manner (Fig. 5 and Supplementary Tables 2-4). One possible reason is that while the mutant used in the study is a double mutant with compromised expression of both the nrpl-1 and $n r p 2-1$ genes, only the level of At NRP1 was elevated in the overexpressor plants. Moreover, the $n r p 1-1$ expression was ectopically regulated in the transgenic plants using an ubiquitously active promoter. Arabidopsis NRP1 and NRP2 functions seems to be only partially redundant, and the genes affected in the single $n r p 1-1$ and nrp2-1 mutant seedlings, respectively, were also found to be different from those deregulated in the double nrp1-1 nrp2-1 mutant ones [1]. These observations support the view that NRPs might control gene expression in a rather unspecific way dependent on their interactions with other factors influenced by their level and/or ratio.

\section{CONCLUSIONS}

Based on the mild phenotypes and the observed gene expression changes associated with altered NRP levels in the mutant/transgenic Arabidopsis plants, one may suppose that although NRPs are not absolutely required for gene regulation, their level needs to be tightly regulated for proper gene expression control. The significant deregulation of photosynthetic, metabolic, and defence genes in parallel with altered growth and developmental rate in the NRP loss-of-function and At NRP1overexpressing plants under short days might be signs of the loss of epigenetic coordination during the adaptation to this suboptimal growth condition.

\section{Acknowledgement}

We are very grateful for Dr. Yan Zhu (State Key Laboratory of Genetic Engineering, Institute of Plant Biology, School of Sciences, Fudan University, Shanghai, China) and Dr. Valerie Frankard (CropDesign N.V., Nevele, Belgium) for the seeds of the nrp1-1 nrp2-1 and the 
This is the author's post-print version. The original containing supplementary information is avalable from Elsewire: https://doi.org/10.1016/j.plantsci.2017.11.006

nrplox Arabidopsis lines, respectively. This work was supported by grants from the Hungarian Ministry for National Economy GINOP-2.3.2-15-2016-00001 and GINOP-2.3.215-2016-00039, and the Hungarian Scientific Research Fund (OTKA K108802; OTKA K83615). IN was supported by the János Bólyai Research Scholarship of the Hungarian Academy of Sciences.

\section{References}

[1] Y. Zhu, A. Dong, D. Meyer, O. Pichon, J. Renou, K. Cao, W.-H. Shen, Arabidopsis NRP1 and NRP2 encode histone chaperones and are required for maintaining postembryonic root growth, Plant Cell 18 (2006) 2879-2892.

[2] J.D. McGhee, G. Felsenfeld, Nucleosome structure, Annu. Rev. Biochem. 49 (1980) 1115-1156.

[3] P. Tessarz, T. Kouzarides, Histone core modifications regulating nucleosome structure and dynamics, Nat. Rev. Mol. Cell Biol. 15 (2014) 703-708.

[4] A. Loyola, G. Almouzni, Histone chaperones, a supporting role in the limelight, Biochim. Biophys. Acta. 1677 (2004) 3-11.

[5] Y. Park, K. Luger, Structure and function of nucleosome assembly proteins, Biochem. Cell Biol. 84 (2006) 549-558.

[6] J. Bíró, I. Farkas, M. Domoki, K. Ötvös, S. Bottka, V. Dombrádi, A. Fehér, The histone phosphatase inhibitory property of plant nucleosome assembly protein-related proteins (NRPs), Plant Physiol. Biochem. 52 (2012) 162-168.

[7] A.K. Tripathi, K. Singh, A. Pareek, S.L. Singla-Pareek, Histone chaperones in Arabidopsis and rice: genome-wide identification, phylogeny, architecture and transcriptional regulation, BMC Plant Biol. 15 (2015) 42.

[8] W. Zhou, Y. Zhu, A. Dong, W.H. Shen, Histone H2A/H2B chaperones: From molecules to chromatin-based functions in plant growth and development, Plant J. 83 (2015) 78-95.

[9] A. Dong, Y. Zhu, Y. Yu, K. Cao, C. Sun, W.-H. Shen, Regulation of biosynthesis and intracellular localization of rice and tobacco homologues of nucleosome assembly protein 1 , Planta 216 (2003) 561-570. 
This is the author's post-print version. The original containing supplementary information is avalable from Elsewire: https://doi.org/10.1016/j.plantsci.2017.11.006

[10] A. Dong, Z. Liu, Y. Zhu, F. Yu, Z. Li, K. Cao, W.-H.H. Shen, Interacting proteins and differences in nuclear transport reveal specific functions for the NAP1 family proteins in plants, Plant Physiol. 138 (2005) 1446-1456.

[11] Z. Liu, Y. Zhu, J. Gao, F. Yu, A. Dong, W.-H. Shen, Molecular and reverse genetic characterization of NUCLEOSOME ASSEMBLY PROTEIN1 (NAP1) genes unravels their function in transcription and nucleotide excision repair in Arabidopsis thaliana, Plant J. 59 (2009) 27-38.

[12] F. Ayaydin, J. Bíró, M. Domoki, G. Ferenc, A. Fehér, Arabidopsis NAP-related proteins ( NRPs ) are soluble nuclear proteins immobilized by heat, Acta Physiol. Plant. 37 (2015) 3.

[13] J. Gao, Y. Zhu, W. Zhou, J. Molinier, A. Dong, W.-H. Shen, NAP1 family histone chaperones are required for somatic homologous recombination in Arabidopsis, Plant Cell 24 (2012) 1437-1447.

[14] W. Zhou, J. Gao, J. Ma, L. Cao, C. Zhang, Y. Zhu, A. Dong, W.H. Shen, Distinct roles of the histone chaperones NAP1 and NRP and the chromatin-remodeling factor INO80 in somatic homologous recombination in Arabidopsis thaliana, Plant J. 88 (2016) 397-410.

[15] Y. Zhu, L. Rong, Q. Luo, B. Wang, N. Zhou, Y. Yang, C. Zhang, H. Feng, L. Zheng, W.-H. Shen, J. Ma, A. Dong, The histone chaperone NRP1 interacts with WEREWOLF to activate GLABRA2 in Arabidopsis root hair development, Plant Cell 29 (2017) 260-276.

[16] K. González-Arzola, A. Díaz-Quintana, F. Rivero-Rodríguez, A. Velázquez-Campoy, M.A. De la Rosa, I. Díaz-Moreno, Histone chaperone activity of Arabidopsis thaliana NRP1 is blocked by cytochrome c, Nucleic Acids Res. 45 (2017) 2150-2165.

[17] L. Selth, J.Q. Svejstrup, Vps75, a new yeast member of the NAP histone chaperone family, J. Biol. Chem. 282 (2007) 12358-12362.

[18] J. Biró, M. Domoki, A. Fehér, NAP-related protein 1 (Atnrp 1) overexpression increases the heat tolerance of Arabidopsis cells/plantlets, J. Plant Biochem. Physiol. 1 (2013) 115.

[19] B. Barna, L. Ádám, Z. Király, Juvenility and resistance of a superoxide-tolerant plant to diseases and other stresses, Naturwissenschaften 80 (1993) 420-422.

[20] B. Barna, A.C. Smigocki, J.C. Baker, Transgenic production of cytokinin suppresses bacterially induced hypersensitive response symptoms and increases antioxidative enzyme levels in Nicotiana spp, Phytopathology. 98 (2008) 1242-1247. 
This is the author's post-print version. The original containing supplementary information is avalable from Elsewire: https://doi.org/10.1016/j.plantsci.2017.11.006

[21] M.D. Robinson, D.J. McCarthy, G.K. Smyth, edgeR: A Bioconductor package for differential expression analysis of digital gene expression data, Bioinformatics 26 (2009) 139-140.

[22] M.D. Robinson, A. Oshlack, A scaling normalization method for differential expression analysis of RNA-seq data, Genome Biol. 11 (2010) R25.

[23] M.E. Ritchie, B. Phipson, D. Wu, Y. Hu, C.W. Law, W. Shi, G.K. Smyth, limma powers differential expression analyses for RNA-sequencing and microarray studies, Nucleic Acids Res. 43 (2015) e47.

[24] Z. Du, X. Zhou, Y. Ling, Z. Zhang, Z. Su, agriGO: A GO analysis toolkit for the agricultural community, Nucleic Acids Res. 38 (2010) W64-W70.

[25] D. Vermaak, Maintenance of chromatin states: an open-and-shut case, Curr. Opin. Cell Biol. 15 (2003) 266-274.

[26] S. Henikoff, Nucleosome destabilization in the epigenetic regulation of gene expression, Nat. Rev. Genet. 9 (2008) 15-26.

[27] E. Bernstein, S.B. Hake, The nucleosome: a little variation goes a long way, Biochem. Cell Biol. 84 (2006) 505-517.

[28] F. Parcy, Flowering: A time for integration, Int. J. Dev. Biol. 49 (2005) 585-593.

[29] S. Yu, V.C. Galvão, Y.-C. Zhang, D. Horrer, T.-Q. Zhang, Y.-H. Hao, Y.-Q. Feng, S. Wang, M. Schmid, J.-W. Wang, Gibberellin regulates the Arabidopsis floral transition through miR156-targeted SQUAMOSA promoter binding-like transcription factors, Plant Cell 24 (2012) 3320-3332.

[30] J.H. Jung, Y. Ju, P.J. Seo, J.H. Lee, C.M. Park, The SOC1-SPL module integrates photoperiod and gibberellic acid signals to control flowering time in Arabidopsis, Plant J. 69 (2012) 577-588.

[31] T. Kania, D. Russenberger, S. Peng, K. Apel, S. Melzer, FPF1 promotes flowering in Arabidopsis, Plant Cell 9 (1997) 1327-1338.

[32] S. Melzer, G. Kampmann, J. Chandler, K. Apel, FPF1 modulates the competence to flowering in Arabidopsis, Plant J. 18 (1999) 395-405.

[33] A. Dutta, P. Choudhary, J. Caruana, R. Raina, JMJ27, an Arabidopsis H3K9 histone demethylase, modulates defense against Pseudomonas syringae and flowering time, Plant J. 38 (2017) 42-49. 
This is the author's post-print version. The original containing supplementary information is avalable from Elsewire: https://doi.org/10.1016/j.plantsci.2017.11.006

[34] E. Breeze, E. Harrison, S. McHattie, L. Hughes, R. Hickman, C. Hill, S. Kiddle, Y. -s. Kim, C.A. Penfold, D. Jenkins, C. Zhang, K. Morris, C. Jenner, S. Jackson, B. Thomas, A. Tabrett, R. Legaie, J.D. Moore, D.L. Wild, S. Ott, D. Rand, J. Beynon, K. Denby, A. Mead, V. Buchanan-Wollaston, High-resolution temporal profiling of transcripts during Arabidopsis leaf senescence reveals a distinct chronology of processes and regulation, Plant Cell 23 (2011) 873-894.

[35] B. Barna, B. Györgyi, Resistance of young versus old tobacco leaves to necrotrophs, fusaric acid, cell wall-degrading enzymes and autolysis of membrane lipids, Physiol. Mol. Plant Pathol. 40 (1992) 247-257.

[36] J. Glazebrook, Contrasting mechanisms of defense against biotrophic and necrotrophic pathogens, Annu. Rev. Phytopathol. 43 (2005) 205-227.

[37] B. Barna, J. Fodor, B.D. Harrach, M. Pogány, Z. Király, The Janus face of reactive oxygen species in resistance and susceptibility of plants to necrotrophic and biotrophic pathogens, Plant Physiol. Biochem. 59 (2012) 37-43.

[38] M. Pogány, J. Koehl, I. Heiser, E.F. Elstner, B. Barna, Juvenility of tobacco induced by cytokinin gene introduction decreases susceptibility to Tobacco necrosis virus and confers tolerance to oxidative stress, Physiol. Mol. Plant Pathol. 65 (2004) 39-47.

[39] B. Becker, S. Holtgrefe, S. Jung, C. Wunrau, A. Kandlbinder, M. Baier, K.-J. Dietz, J.E. Backhausen, R. Scheibe, Influence of the photoperiod on redox regulation and stress responses in Arabidopsis thaliana L. (Heynh.) plants under long- and short-day conditions, Planta 224 (2006) 380-393.

[40] S. Li, A. Mhamdi, A. Trotta, S. Kangasjärvi, G. Noctor, The protein phosphatase subunit PP2A-B' $\gamma$ is required to suppress day length-dependent pathogenesis responses triggered by intracellular oxidative stress, New Phytol. 202 (2014) 145-160.

[41] G. Queval, E. Issakidis-Bourguet, F.A. Hoeberichts, M. Vandorpe, B. Gakière, H. Vanacker, M. Miginiac-Maslow, F. Van Breusegem, G. Noctor, Conditional oxidative stress responses in the Arabidopsis photorespiratory mutant cat 2 demonstrate that redox state is a key modulator of daylength-dependent gene expression, and define photoperiod as a crucial factor in the regulation of $\mathrm{H}_{2} \mathrm{O}_{2}$-induced cell death, Plant J. 52 (2007) 640-657.

[42] G. Queval, J. Neukermans, S. Vanderauwera, F. van Breusegem, G. Noctor, Day length is a key regulator of transcriptomic responses to both $\mathrm{CO}_{2}$ and $\mathrm{H}_{2} \mathrm{O}_{2}$ in Arabidopsis, Plant, Cell Environ. 35 (2012) 374-387. 
This is the author's post-print version. The original containing supplementary information is avalable from Elsewire: https://doi.org/10.1016/j.plantsci.2017.11.006

[43] L. Michelet, A. Krieger-Liszkay, Reactive oxygen intermediates produced by photosynthetic electron transport are enhanced in short-day grown plants, Biochim. Biophys. Acta - Bioenerg. 1817 (2012) 1306-1313.

[44] A.A. Dghim, A. Mhamdi, M.N. Vaultier, M.P. Hasenfratz-Sauder, D. Le Thiec, P. Dizengremel, G. Noctor, Y. Jolivet, Analysis of cytosolic isocitrate dehydrogenase and glutathione reductase 1 in photoperiod-influenced responses to ozone using Arabidopsis knockout mutants, Plant, Cell Environ. 36 (2013) 1981-1991.

[45] Y. Shen, E. Issakidis-Bourguet, D.X. Zhou, Perspectives on the interactions between metabolism, redox, and epigenetics in plants, J. Exp. Bot. 67 (2016) 5291-5300.

[46] M. Rahikainen, J. Pascual, S. Alegre, G. Durian, S. Kangasjärvi, PP2A phosphatase as a regulator of ROS signaling in plants, Antioxidants 5 (2016) 8.

[47] B. Heidari, D. Nemie-Feyissa, S. Kangasjärvi, C. Lillo, Antagonistic regulation of flowering time through distinct regulatory subunits of protein phosphatase $2 \mathrm{~A}$, PLoS One 8 (2013) e67987.

[48] A. Trotta, M. Wrzaczek, J. Scharte, M. Tikkanen, G. Konert, M. Rahikainen, M. Holmstrom, H.-M. Hiltunen, S. Rips, N. Sipari, P. Mulo, E. Weis, A. von Schaewen, E.-M. Aro, S. Kangasjarvi, Regulatory subunit B' $\gamma$ of protein phosphatase 2A prevents unnecessary defense reactions under low light in Arabidopsis, Plant Physiol. 156 (2011) 1464-1480.

[49] J. V Kus, K. Zaton, R. Sarkar, R.K. Cameron, Age-related resistance in Arabidopsis is a developmentally regulated defense response to Pseudomonas syringae, Plant Cell 14 (2002) 479-490.

[50] J. Iglesias, M. Trigueros, M. Rojas-Triana, M. Fernández, J.P. Albar, R. Bustos, J. Paz-Ares, V. Rubio, Proteomics identifies ubiquitin-proteasome targets and new roles for chromatin-remodeling in the Arabidopsis response to phosphate starvation, J. Proteomics 94 (2013) 1-22.

[51] S. Pape, C. Thurow, C. Gatz, The Arabidopsis PR-1 promoter contains multiple integration sites for the coactivator NPR1 and the repressor SNI1, Plant Physiol. 154 (2010) 1805-1818.

[52] R.A. Mosher, W.E. Durrant, D. Wang, J. Song, X. Dong, A comprehensive structurefunction analysis of Arabidopsis SNI1 defines essential regions and transcriptional repressor activity, Plant Cell 18 (2006) 1750-1765. 
This is the author's post-print version. The original containing supplementary information is avalable from Elsewire: https://doi.org/10.1016/j.plantsci.2017.11.006

[53] W.E. Durrant, S. Wang, X. Dong, Arabidopsis SNI1 and RAD51D regulate both gene transcription and DNA recombination during the defense response, Proc. Natl. Acad. Sci. U. S. A. 104 (2007) 4223-4227. 
This is the author's post-print version. The original containing supplementary information is avalable from Elsewire: https://doi.org/10.1016/j.plantsci.2017.11.006

\section{Figure legends}

Fig. 1. Response to fungal infection of wild type (wt), nrp1-1 nrp2-1 double mutant (nrpmut) and At NRP1 overexpressing (nrp1ox) Arabidopsis plants grown under short day conditions (11h light $13 \mathrm{~h}$ dark). A) lon leakage from control and powdery mildew (Golovinomyces orontii)-infected Arabidopsis leaves as measured by conductivity $\left(\mu \mathrm{S} g\right.$ fresh mass $\left.{ }^{-1}\right)$ test. B) Disease development on Arabidopsis leaves measured by diameter $(\mathrm{mm})$ of necrotic spots after Sclerotinia sclerotiorum infection. C) Representative images of Sclerotinia-treated leaves with necrotic regions indicated by arrowheads D) Ion leakage from control and Sclerotinia-infected Arabidopsis leaves as measured by conductivity ( $\mu \mathrm{S}$ g fresh mass $\left.{ }^{-1}\right)$ test.

In one experiment, five-five whole control or infected wild type, mutant, and overexpressor leaves were randomly taken in three repetitions from at least six - six plants. Mean values \pm SD of a representative experiment are shown. The experiments were independently repeated five (powdery mildew) and three (Sclerotinia) times with similar results.

Fig. 2. Response to bacterial infection of wild type (wt), nrp1-1 nrp2-1 double mutant (nrpmut) and At NRP1 overexpressing (nrp1ox) Arabidopsis plants grown under short day conditions (11h light 13h dark). A) lon leakage from control, Pseudomonas syringae pv. tomato DC3000 (DC3000), or Pseudomonas syringae pv. syringae strain 61 (Ps61) infected Arabidopsis leaves as measured by conductivity ( $\mu \mathrm{S}$ g fresh mass ${ }^{-1}$ ) tests 72 hours after infection. B) Growth of Pseudomonas syringae pv. tomato DC3000 (DC3000), or Pseudomonas syringae pv. syringae strain 61 (Ps61) in infected Arabidopsis leaves as measured by bacterial concentration (cfu $\times 10^{3} \mathrm{ml}^{-1}$ ) 72 hours after infection. Inoculation was carried out by brushing the leaves of 40-days-old plant either with water (control) or with bacterial suspension $\left(10^{8} \mathrm{cfu} \mathrm{cm}^{-3}\right)$. 
This is the author's post-print version. The original containing supplementary information is avalable from Elsewire: https://doi.org/10.1016/j.plantsci.2017.11.006

Mean values \pm SD of three replicates of a representative experiment are shown. The experiments were independently repeated five (ion leakage) or four (bacterial concentration) times with similar results.

Fig. 3. The NRP protein(s) affects plant growth under short day conditions. Representative pictures (A) and the development of the rosette diameter (B) of wild type (wt), nrp 1-1 nrp 2-1 double mutant (nrpmut) and the At NRP1 overexpressing (nrp1ox) Arabidopsis plants ( $n=15)$ grown under short day conditions (8h light/16h dark) for the indicated time periods. Error bars represent standard deviation.

Fig. 4. Bolting time and senescence of wild type (wt), nrp1-1 nrp2-1 double mutant (nrpmut) and At NRP1 overexpressing (nrp1ox) Arabidopsis plants grown under short day conditions. A) Visual evaluation of bolting. The number of plants starting to bolt at a given time point is indicated beside the signs that show the average leaf numbers (+- standard errors) at bolting. Data belonging to plants having the same genotype are encircled. B) Representative photos of 93-day-old plants grown under short days. Arrowheads indicate senescing leaves. C) Visual evaluation of leaf senescence of plants grown under short days for 65-107 days. The percentage of leaves with yellow regions (among all leaves of three randomly selected plants) and the percentage of plants with at least one yellow leaf (among 15 investigated plants per genotype) are shown at the indicated time points (averages with standard errors). Different letters mean statistically significant difference $(p<0,05)$ using Duncan's multiple comparison test after ANOVA.

Fig. 5. The Venn diagram of the number of genes the expression of which was significantly altered in 72-days-old nrp 1-1 nrp 2-1 double mutant (nrpmut) and At NRP1 overexpressing ( $n r p 10 x$ ) plants in comparison to the wild type. "up" - at least 2-fold upregulated in respect of wild type expression; "down" - at least 2-fold downregulated in respect of wild type expression. 
This is the author's post-print version. The original containing supplementary information is avalable from Elsewire: https://doi.org/10.1016/i.plantsci.2017.11.006

Fig. 6. Comparison of the relative expression data of selected senescence and/or pathogenesis associated genes obtained by transcriptome sequencing (RNA-Seq; two biological replicates) and realtime quantitative PCR (QPCR; two biological and three technical replicates). Averages and standard deviations are shown. The expression of the glutathione S-transferase TAU 1 (AT2G29490) was used as a normalization factor and the expression in the wild type was used as a reference (1 unit). 

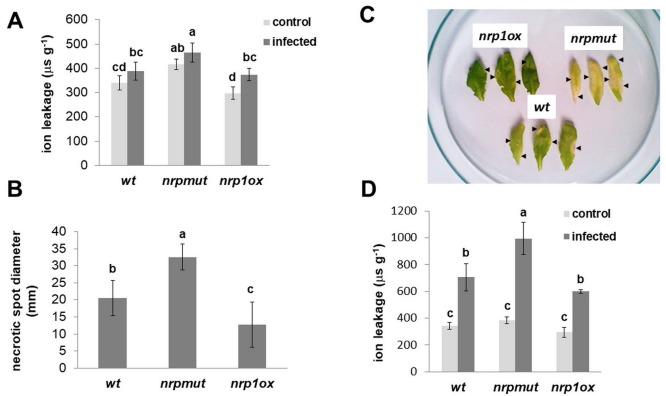


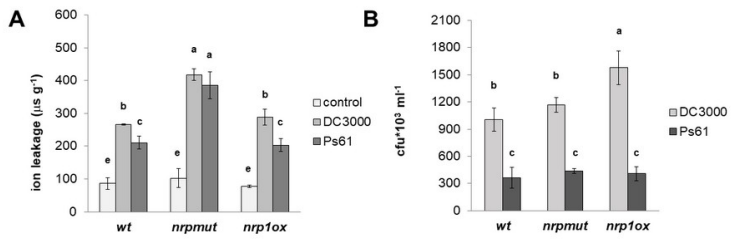


A

wt
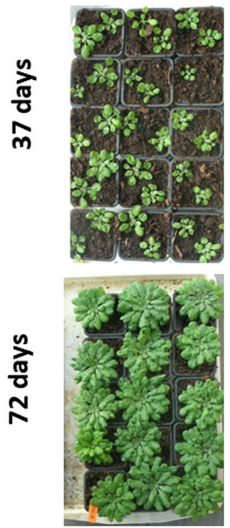

nrpmut
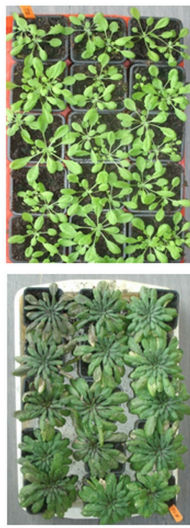

nrp1ox
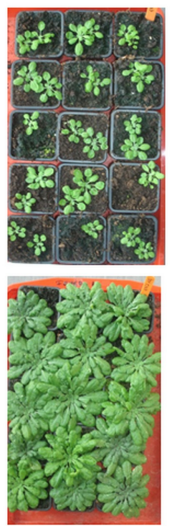

B

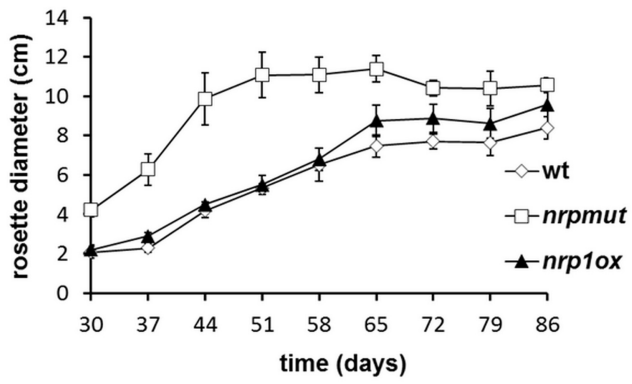




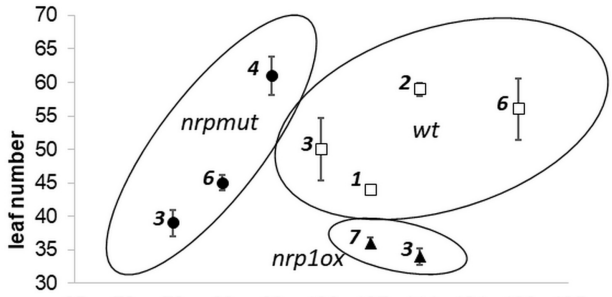

$\begin{array}{lllllllllll}65 & 72 & 79 & 86 & 93 & 100 & 107 & 114 & 121 & 128 & 135\end{array}$ bolting time (days)

口wt $\bullet$ nrpmut $\Delta$ nrp1ox

B
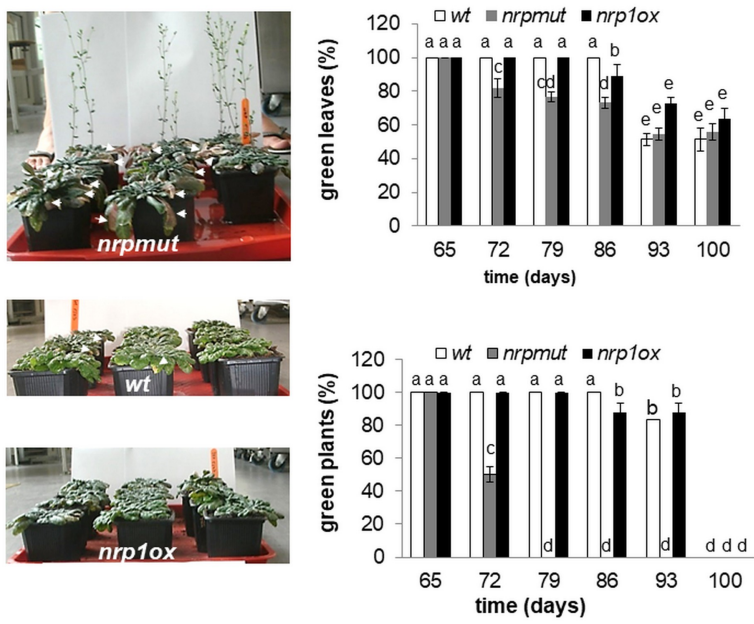


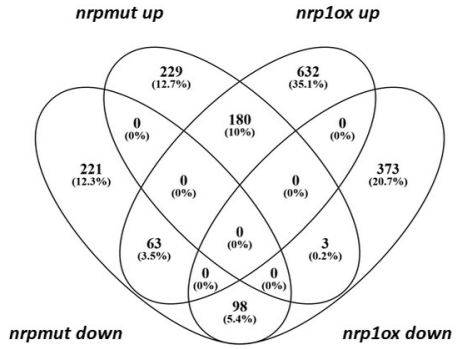



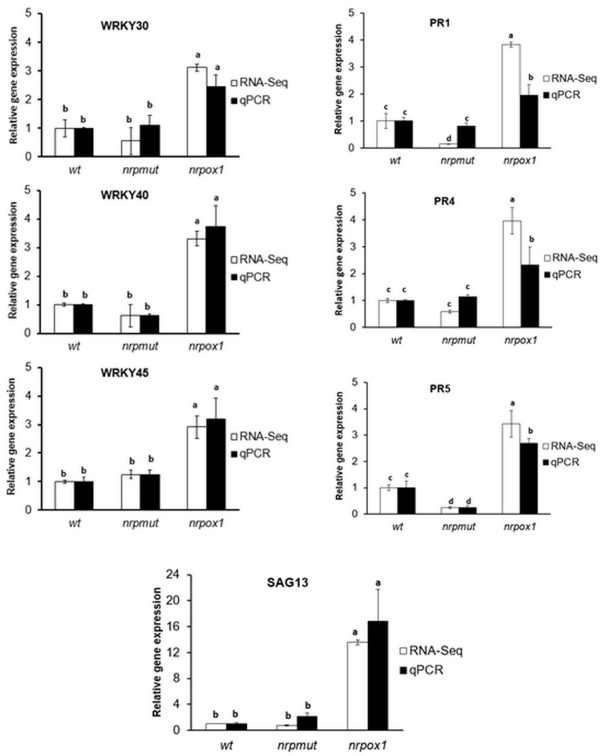


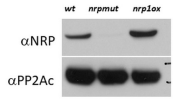

Exp. 1

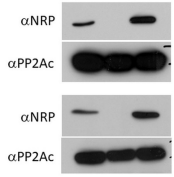

Exp. 2

Exp. 3

\section{Quantitative analysis}

3

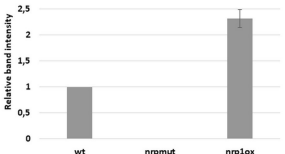

wt

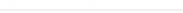

nipmut

nrplox

Supplementary Fig. 1. Abundance of the At NRP proteins in the wild type (wt), the nrp 1-1 nrp 2-1 mutant (nrpmut) and the At NRP1 overexpressing (nrp1ox) Arabidopsis plants as determined by Western analysis in three biologically independent experiments (for technical details see Bíró et al. 2012). The level of the PP2A catalytic subunit was used as sample loading control. The histogram shows the average and standard deviaton of relative band intensities as measured by the Fiji software (Schindelin, J.; Arganda-Carreras, I. \& Frise, E. et al. (2012), "Fiji: an open-source platform for biological-image analysis", Noture methods 9(7):676-682). The band intensities of NRPs were normalised by the PP2Ac intensities and than by the intensity in the wild type (value=1). 

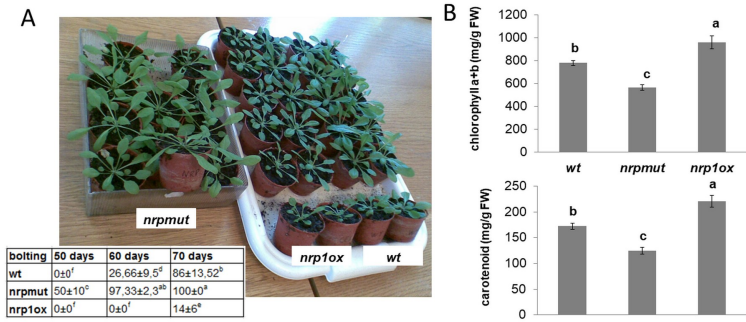

$w t$

nrpmut

nrp1ox

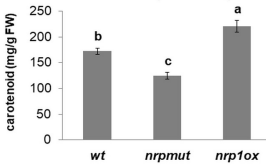

Supplementary Fig. 2. Preliminary observation about the growth differences of the investigated lines. (A) Developmental stage of 40 -days-0id wid type (WT, two rows on

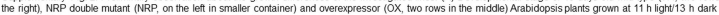
condition. Note the larger size and lighter green color (and initiation of some inflorescence stem) of mutant, and the smaller size and darker green color of overexpressor leaves. The table shows the percentage (\%) of bolting plants between a 50,60 , and 70 days. (B) Photosymthetic pigment content of the leaves of the plants shown on (A) Different letters mean statistically signicant difference at the $p=0,05$ level using Duncan's test. 


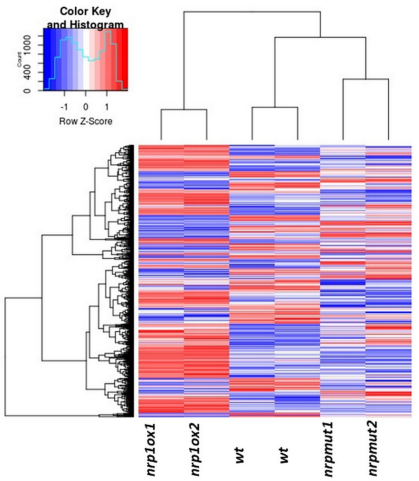

Supplementary Fig. 3. The result of unsupervised hierarchical clustering of whole RNAseq profiles as a heat map 


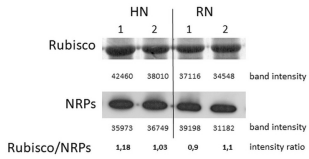

Supplementary Fig. 4. At NRP levels in the leaves of plants just entering to the bolting phase under long (16h light/8h dark);HN) or short ( 8 h light, 16 h dark;RN) day conditions. Proteins were isolated from plants grown in two separate expreiments ( 1 and 2). The level of At NRPs was determined by Western anlysis using $\alpha$ NRP1 antibody (lowere lane) and was compared to that of the Rubisco large subunit protein visualised by Ponceau staining on the PVDF membrane used for the Western blot (upper lane). Band intensities were determined by the Fiji software (Schindelin, J.; Arganda-Carreras, I. \& Frise, E. et al. (2012), "Fiji: an open-source platform for biological-image analysis", Noture methods 9(7): 676-682). 